\title{
Reversible, Time-Dependent Inhibition of CYP3A-Mediated Metabolism of Midazolam and Tacrolimus by Telaprevir in Human Liver Microsomes
}

\author{
Brian Chapron, ${ }^{1}$ Linda Risler, ${ }^{1}$ Brian Phillips, ${ }^{1}$ Carol Collins, ${ }^{1}$ Kenneth E. Thummel, ${ }^{1}$ and Danny D. Shen ${ }^{1,2}$ \\ ${ }^{1}$ Department of Pharmaceutics, University of Washington, Seattle, Washington \\ ${ }^{2}$ Department of Pharmaceutics, H272 Health Sciences Building, University of Washington, Seattle, WA.
}

Received, January 15, 2015; Revised, January 21, 2015; Accepted, March 19, 2015; Published, March $20,2015$.

\begin{abstract}
Purpose Telaprevir inhibits CYP3A resulting in drug-drug interactions (DDI) of unprecedented magnitude. We investigated the mechanisms by which telaprevir inhibits the oxidation of midazolam and tacrolimus in human liver microsomes (HLM). Methods We performed a static mechanistic DDI prediction to evaluate whether previously reported competitive inhibition of CYP3A by telaprevir and its diastereomeric metabolite - VRT-127394 is sufficient to explain the remarkable reduction in oral clearance observed with oral midazolam and tacrolimus. To further explore the inhibitory mechanisms of telaprevir, we assessed whether telaprevir-mediated inhibition of the oxidation of midazolam and tacrolimus is time-dependent in human liver microsomes, and whether any observed time-dependency was irreversible or reversible in nature. Results The competitive inhibition model failed to account for the magnitude of telaprevir interactions in human subjects. In comparing HLM incubations with and without a prior 30-min exposure to telaprevir, a respective 4- and 11-fold reduction in IC50 was observed with midazolam and tacrolimus as substrates. This time-dependent inhibition was shown to be NADPH-dependent. Upon dilution of microsomes following pre-incubation with telaprevir, time-dependent inhibition of midazolam metabolism was completely reversed, whereas partial reversal occurred with tacrolimus. Conclusions The interaction between telaprevir and midazolam or tacrolimus involves both competitive and time-dependent inhibition. The time-dependent component is not explained by irreversible inactivation of CYP3A. Formation of potent inhibitory metabolites may contribute to the remarkable in vivo inhibitory potency of telaprevir.
\end{abstract}

This article is open to POST-PUBLICATION REVIEW. Registered readers (see "For Readers") may comment by clicking on ABSTRACT on the issue's contents page.

\section{INTRODUCTION}

Introduction of the first hepatitis $\mathrm{C}$ virus (HCV) protease inhibitors, telaprevir and boceprevir, in 2011 marked a major milestone in hepatitis $\mathrm{C}$ drug development. HCV protease inhibitors, when used in combination with ribavirin and peginterferon alpha, were the first of a new generation of directacting drug treatments for combating HCV (1-2). However, during development, telaprevir was found to precipitate a number of drug-drug interactions (DDI) (3). In particular, attention was drawn to the profound interaction between telaprevir and the immunosuppressant, tacrolimus (4). Although newer and more effective drugs have since replaced telaprevir in the treatment of hepatitis $C$, the unprecedented magnitude of this interaction, an approximately 67 -fold reduction in tacrolimus oral clearance, raises interesting scientific questions with respect to the pharmacokinetic mechanism(s) of this interaction (5).

In the "Clinical Pharmacology \& Biopharmaceutics Review" section of the NDA for telaprevir (6), Vertex Pharmaceuticals reported on in vitro studies demonstrating competitive inhibition of CYP3A4, as measured by midazolam 1 '-hydroxylation, with a $K_{i}$ of $1.43 \mu \mathrm{M}$. A major metabolite, VRT-127394, was also identified as a competitive inhibitor of CYP3A4 with a reported $K_{i}$ of $0.94 \mu \mathrm{M}$. In the present study, we attempted a prediction of the joint effects of telaprevir and

Corresponding Author: Danny D Shen, PhD, Department of Pharmaceutics, H272 Health Sciences Building, University of Washington, Seattle, WA 98195-7610, Email: ds@uw.edu 
VRT-127395 based upon a mechanistic static DDI model that assumed reversible, competitive inhibition of CYP3A in the gut mucosa and the liver. The competitive inhibition DDI model underpredicted the observed clinical DDI considerably. This under-prediction was more pronounced in the case of tacrolimus than midazolam. One possible explanation for this discrepancy between the predicted and observed interactions could be the presence of additional mechanisms of CYP3A inhibition. An in vitro maximum inactivation rate constant ( $\mathrm{k}_{\text {inact }}$ ) of $0.065 \mathrm{~min}^{-1}$ using midazolam as the reporter substrate was quoted in the telaprevir NDA (6), implying evidence of mechanism-based inhibition (MBI). More recently, Oda and Yamano reported observing MBI of tacrolimus oxidation by telaprevir in human liver microsomes (HLM) (7).

The purpose of the present study was to confirm and further characterize the putative MBI by telaprevir in vitro. We were able to demonstrate time-dependent inhibition of both midazolam and tacrolimus metabolism in HLM. However, we observed reversibility in the time-dependent inhibition, pointing to time-dependent inhibition mechanisms other than irreversible MBI.

\section{METHODS}

\section{Materials}

Telaprevir was obtained from Toronto Research Chemicals (Toronto, Canada). NADPH, tacrolimus and rapamycin were obtained from Sigma-Aldrich (Steinheim, Germany). 13-O-desmethyltacrolimus (13-DMT) was extracted and purified from incubates of HLMs with tacrolimus (8). Midazolam and 1'-hydroxymidazolam (1'-OH-MDZ) were obtained from Cerilliant (Round Rock, TX). Pooled HLMs were obtained from Xenotech (Lenexa, KS). Recombinant P450s were obtained from BD Biosciences (Woburn, MA). All other solvents and reagents were of analytical reagent grade.

\section{Modeling of Competitive Inhibition Scenarios}

We utilized a mechanistic static DDI modeling approach described in several previous publications (9-11). The fold-change in oral clearance $\left(C L_{\text {oral }}\right)$ of either midazolam or tacrolimus in the presence of telaprevir and VRT-127394 was the principal endpoint for our model prediction. Prediction of $C L_{\text {oral }}$ entailed an assessment of the impact of CYP3A inhibition by telaprevir and VRT-127394 on the fraction absorbed $\left(F_{a}\right)$, fraction escaping first-pass gut metabolism $\left(F_{g}\right)$, fraction escaping first-pass hepatic metabolism $\left(F_{h}\right)$, and systemic clearance $\left(C L_{s y s}\right)$ for midazolam and tacrolimus. Baseline values of $F_{g}, F_{h}$ and $C L_{h}$ (i.e., in the absence of inhibitors) were either taken directly or estimated based upon data from the literature (12, 13). Linear pharmacokinetics were assumed for both object drugs. The following sections provide salient details on how the $F_{a}, F_{g}, F_{h}$ and $C L_{h}$ parameters during telaprevir co-administration were computed.

Systemic Clearance $\left(\boldsymbol{C} \boldsymbol{L}_{s y s}\right)$. Systemic elimination of both midazolam and tacrolimus was assumed to occur exclusively via hepatic metabolism; hence, $C L_{s y s}$ was set equal to hepatic clearance $\left(C L_{h}\right)$. Baseline hepatic intrinsic clearance $\left(C L_{h, \text { int }}\right)$ of midazolam or tacrolimus was estimated from their corresponding literature-reported systemic clearance and hepatic blood-flow $\left(Q_{h}\right)$ according to the well-stirred hepatic clearance model.

All clearance values were referenced to blood concentrations of the object drug. Specifically, plasma clearance values for midazolam were converted to blood clearance values assuming an equilibrium blood-to-plasma ratio of 0.66 for midazolam (14). $Q_{h}$ was assumed to be $90 \mathrm{~L} / \mathrm{h}$ (15).

$$
C L_{h}=\frac{Q_{h} * C L_{h \text { int }}}{Q_{h}+C L_{h \text { int }}}
$$

A term reflecting the fold-reduction in the intrinsic clearance parameter for the object drug due to additive competitive inhibition by telaprevir and VRT-127394 was defined as the inhibition ratio or $I R$ (10). Subscripts in the $I R$ parameter denote the assumptions adopted regarding inhibitor concentrations at the clearance site; viz., inhibition driven by systemic inhibitor concentration $\left(I R_{s y s}\right)$, and first-pass extraction inhibited by local gut mucosal concentration $\left(I R_{g, \max }\right)$ or hepatic portal vein concentration $\left(I R_{h p v, \max }\right)$ during oral absorption of the inhibitor. For example, $I R_{s y s}$ denotes the inhibition ratio for intrinsic clearance using unbound circulating concentrations of telaprevir (Isys,telaprevir $)$ and VRT-127394 (I sys,VRT127394 $)$.

$I R_{\text {sys }}=\left(1+\frac{f_{u} * I_{\text {sys, telaprevir }}}{K_{i, \text { tel coprevir }}}+\frac{f_{u} * I_{\text {sys,VRT } 127394}}{K_{i, \text { VRT } 127394}}\right)$ 
Only unbound telaprevir and VRT-127394 in plasma were assumed to access the intrahepatocellular site and inhibit CYP3A.

Plasma concentrations of telaprevir and VRT127394 during co-administration with each object drug were taken from their respective DDI studies in healthy volunteers $(5,12)$. Telaprevir and VRT127394 concentrations were evaluated as a range from the lower to upper bound of the reported 95\% confidence interval of their average steady-state plasma concentrations during chronic dosing at 750 mg of telaprevir every 8 hours. The unbound inhibitor concentrations were estimated by multiplying the total concentrations by the fraction unbound $\left(f_{u}\right)$ of 0.24 to 0.41 , as reported in the telaprevir NDA (6). Competitive inhibition constants of 1.43 and $0.94 \mu \mathrm{M}$ were assigned for telaprevir $\left(K_{i, \text { telaprevir }}\right)$ and VRT-127394 $\left(K_{i, \text { VRT127394 }}\right)$ respectively, as reported in the NDA (6).

As indicated in the equation below, $I R_{s y s}$ was only applied to the CYP3A-mediated fraction of intrinsic clearance $\left(f_{m, C Y P 3 A}: 0.94\right.$ for midazolam and 1.0 for tacrolimus), thereby accounting for hepatic metabolism that is not inhibited by telaprevir (i.e., minor non-CYP3A mediated pathways) (16). The assumption of complete metabolism of tacrolimus by CYP3A (i.e., $f_{m, C Y P 3 A}=1$ ) was substantiated by conducting substrate depletion experiments with recombinant CYP1A1, CYP1A2, CYP2B6, CYP2C19, CYP2C8, CYP2C9, CYP2D6, CYP3A4 and CYP3A5. In this experiment, the only P450s that observably metabolized tacrolimus were CYP2D6, CYP3A4 and CYP3A5. The intrinsic clearance per pmol of CYP2D6 was approximately one-third that of CYP3A (data not shown), or 60fold lower when scaled to their respective enzyme content in the liver (17). Therefore, CYP2D6mediated metabolism of tacrolimus was ignored in our modeling on the basis of its modest activity and low expression of CYP2D6 in both the gut and liver (17). Furthermore, adopting an $f_{m, C Y P 3 A}=1$ for tacrolimus afforded the fullest potential in predicting the magnitude of competitive inhibition.

$C L_{\text {h.int, inh }}=\frac{C L_{\text {h.int }} * f_{m, C Y P 3 A}}{I R_{s y s}}+C L_{\text {h.int }} *\left(1-f_{m, C Y P 3, A}\right)$ (3)

Hepatic Clearance $\left(\boldsymbol{C} \boldsymbol{L}_{h}\right)$. Hepatic intrinsic clearance with competitive inhibition accounted for $\left(C L_{h}\right.$, int,inh $)$ was applied to the well-stirred model to generate an estimate for the inhibited systemic hepatic clearance $\left(C L_{h, i n h}\right)(15)$.

Gut Absorption $\left(\boldsymbol{F}_{\boldsymbol{a}}\right)$. Drug release from the dosage form and mucosal permeability were assumed not to be affected by telaprevir; i.e., $F_{a}$ remains the same in the presence of the inhibitor. This assumption ignores the potential effect of intestinal P-glycoprotein on the intestinal absorption of tacrolimus. The values for $F_{a}$ were calculated from the literature and were 0.90 and 0.96 for midazolam and tacrolimus respectively (13, 18).

Gut First-Pass $\left(\boldsymbol{F}_{\boldsymbol{g}}\right)$. The usual challenge in the static prediction of first-pass inhibition is what relevant inhibitor concentration ought to be used to calculate the $I R$ term. We chose to model both the "least" and "maximum" impact scenarios; the former represents the period after the intestinal absorption of telaprevir had been completed, whereas the latter considers the period shortly after inhibitor co-administration. The following equation based upon the $\mathrm{Q}_{\text {gut }}$ model was used to assess the "least" scenario; that is, the impact of average systemic inhibitor concentrations on the fraction of drug escaping first-pass intestinal extraction $\left(F_{g, i n h}\right)$ (10). Intestinal metabolism of midazolam and tacrolimus was assumed to be solely mediated by mucosal CYP3A.

$$
F_{g, i n h}=\frac{F_{g}}{F_{g}+\left(1-F_{g}\right) *\left(\frac{1}{I R_{s y s}}\right)}
$$

We also estimated the fraction escaping intestinal extraction for the "maximum" inhibition scenario $\left(F_{g, \text { inh }}^{\prime}\right)$; in this case, the first-order absorption rate constant $\left(k_{a}\right)$, telaprevir dose $(D)$, and blood drainage from the intestinal villi $\left(Q_{\text {villi }}\right)$ (19) determine the local (mucosal) concentration of telaprevir $\left(I_{g, \max }\right)$ and inhibition ratio $\left(I R_{g, \max }\right)$. Note that circulating concentration of VRT-127394 continues to apply in this scenario.

$$
I_{g, \max }=\frac{k_{a} * F_{a} * D}{Q_{\text {villi }}}
$$

$$
I R_{g, \max }=1+\frac{I_{g, \max }+\left(f_{u} * I_{s y s, \text { telaprevir }}\right)}{K_{i, \text { telaprevir }}}+\frac{f_{u} * I_{s y s, V R T 127394}}{K_{i, V R T 127394}}
$$


Hepatic First-Pass $\left(\boldsymbol{F}_{\boldsymbol{h}}\right)$. The previously derived $C L_{h, i n h}$ was used to predict the fraction escaping hepatic first-pass metabolism in a "least" inhibition scenario $\left(F_{h, i n h}\right)$, wherein the effective concentrations of the inhibitors equal their systemic concentrations during the post-absorptive phase of telaprevir pharmacokinetics.

$$
F_{h, i n h}=1-\left(\frac{C L_{h, i n h}}{Q_{h}}\right)
$$

The following equation represents the "maximum" inhibition scenario during first-pass following simultaneous telaprevir and object drug administration. This scenario assumes a transiently elevated inflow portal venous concentration that equals the sum of re-circulating and newly absorbed telaprevir from the gut lumen into the hepatoportal blood flow $\left(I_{h p v, \max }\right)(10)$.

$$
\begin{aligned}
& I_{h y v \max }=\frac{k_{a} * F_{a} * F_{g} * D}{Q_{h}} \\
& I R_{\text {hyv, max }}=1+\frac{f_{u} *\left(I_{\text {hpv, max }}+I_{\text {sys, telaprevir }}\right)}{K_{i, t \text { lela previr }}}+\frac{f_{u} * I_{\text {sys,VRT } 127394}}{K_{i, V R T 127394}}
\end{aligned}
$$

Again, hepatoportal concentrations of VRT127394 were assumed to be equal to systemic concentrations (i.e., no sequential first pass). The above $I R_{h p v, \max }$ expression applies if the entire telaprevir dose was absorbed instantly at the same time as the object drug. It allows us to determine the effect of these hepatoportal concentrations on the fraction of substrate escaping first-pass metabolism under a theoretical "maximum" inhibition conditions $\left(F^{\prime}{ }_{h, i n h}\right)$.

Systemic bioavailability of each object drug was computed in the absence $\left(F_{\text {total }}=F_{a} \cdot F_{g} \cdot F_{h}\right)$ and presence of co-administered inhibitor under either the "least" or "maximum" inhibition scenario $\left(F_{\text {total, inh }}=F_{a} \cdot F_{g, \text { inh }} \cdot F_{h, \text { inh }}\right.$ or $F_{\text {total,inh }}^{\prime}=$ $\left.F_{a} \cdot F^{\prime}{ }_{g, i n h} \cdot F^{\prime}{ }_{h, i n h}\right)$. A third "extreme" scenario was considered that assumed complete absorption $\left(F_{a}=\right.$ 1) and total abolition of first-pass elimination processes $\left(F_{g, i n h} \cdot F_{h, i n h}=1\right)$. Essentially, this final scenario is one where systemic availability $\left(F_{\text {total,inh }}\right)$ is set to a fixed value of 1 and oral clearance $\left(C L_{\text {oral,inh }}\right)$ is effectively reduced to the inhibited systemic or hepatic clearance $\left(C L_{s y s, i n h}\right.$ or $\left.C L_{h, i n h}\right)$.
All three scenarios were evaluated in terms of the predicted fold-reduction in oral clearance from telaprevir co-administration compared to the uninhibited state. Below are the final equations used to determine the fold-reduction from the baseline oral clearance to that in the "least" $\left(C L_{\text {oral in }}\right)$, "maximum" $\left(C L\right.$ ' $\left.{ }_{\text {oral }, \text { inh }}\right)$, and "extreme" scenarios respectively.

$$
\begin{aligned}
& \frac{C L_{\text {oral }}}{C L_{\text {oral in h }}}=\frac{C L_{\text {oral }}}{\left(\frac{C L_{\text {sys, inh }}}{F_{a} * F_{\text {g,inh }} * F_{h, \text { inh }}}\right)} \\
& \frac{C L_{\text {oral }}}{C L_{\text {oral in } h}}=\frac{C L_{\text {oral }}}{\left(\frac{C L_{\text {sys, in } h}}{F_{\text {a }} * F_{\text {g,in } h} * F_{h, \text { in } h}}\right)} \\
& \frac{C L_{\text {oral }}}{C L_{\text {oral in h }}}=\frac{C L_{\text {oral }}}{\left(\frac{C L_{\text {sys, inh }}}{1}\right)}
\end{aligned}
$$

\section{IC50 Shift Experiments}

Incubation mixtures (200 $\mu 1$ volume), containing $0.05 \mathrm{mg} / \mathrm{ml}$ pooled HLM, fixed concentration of substrate (either $1 \mu \mathrm{M}$ midazolam or $0.5 \mu \mathrm{M}$ tacrolimus), and variable concentration of telaprevir or corresponding vehicle control in a phosphate buffer medium $\left(100 \mathrm{mM} \mathrm{KK_{2 }} \mathrm{PO}_{4}, 1 \mathrm{mM}\right.$ EDTA, $\mathrm{pH}=7.4$ ), were pre-warmed at $37^{\circ} \mathrm{C}$ in a shaker water bath for $5 \mathrm{~min}$ prior to initiation of reaction. To construct an IC50 curve, the telaprevir concentration was varied from $0.05 \mu \mathrm{M}$ to $10 \mu \mathrm{M}$. Due to non-specific binding of tacrolimus to the HLM and incubation vessel, the unbound concentration in the $0.5 \mu \mathrm{M}$ tacrolimus incubations was effectively $0.1 \mu \mathrm{M}$ in this particular experimental setup. Midazolam and telaprevir had no appreciable $(<20 \%)$ non-specific binding and thus their nominal concentrations were considered as the unbound concentrations (20). The concentrations of midazolam or tacrolimus were chosen so as to achieve unbound concentrations below their respective $K_{m}$ for CYP3A (20-21). The CYP-mediated reaction was initiated with $50 \mu$ of $5 \mathrm{mM}$ NADPH (final $1 \mathrm{mM}$ concentration) and quenched with either $250 \mu \mathrm{l}$ of ice-cold acetonitrile (midazolam) or $2 \mathrm{ml}$ MTBE (tacrolimus) after 
incubating for 2 minutes. All incubations were conducted in quadruplicates.

For midazolam incubations, $20 \mu 1$ of $\mathrm{d}_{4}-1$ '-OHMDZ $(0.25 \mathrm{ng} / \mu \mathrm{l})$ was added as internal standard to each of the quenched reaction mixtures, which were then centrifuged at $14,000 \mathrm{rpm}$ for $10 \mathrm{~min}$ at $4^{\circ} \mathrm{C}$. A portion of the supernatant was then transferred to 96-well plates for LC/MS-MS analysis. For tacrolimus incubations, $20 \mu \mathrm{l}$ of rapamycin (1.1 $\mathrm{ng} / \mu \mathrm{l})$ was added as internal standard before addition of MTBE. The aqueous phase was frozen by dry ice. The solvent phase was then decanted and evaporated under $\mathrm{N}_{2}$ gas. The sample residues were reconstituted in $75 \mu \mathrm{l}$ of methanol and transferred to glass inserts for LC/MS-MS analysis. Activity of CYP3A was measured by 1'-OH-MDZ formation for midazolam and 13-DMT formation for tacrolimus.

To assess time-dependent inhibition, IC50 experiments were performed after pre-incubating $0.05 \mathrm{mg} / \mathrm{ml} \mathrm{HLM}$ with $1 \mathrm{mM}$ NADPH and varying concentration of telaprevir $(0.05$ to $10 \mu \mathrm{M})$ for 30 minutes. Parallel pre-incubations with vehicle control $(0.3 \%$ DMSO $)$ were also performed to assess any non-telaprevir mediated decreases in CYP3A activity related to the $30 \mathrm{~min}$ preincubation. Substrate reaction was then initiated by the addition of $1 \mu \mathrm{M}$ midazolam or $0.5 \mu \mathrm{M}$ tacrolimus to the reaction mixture. These reactions were quenched and processed for analysis as described above.

\section{NADPH Dependence and Reversibility Experiments}

Co-incubations of substrate $(0.5 \mu \mathrm{M}$ for tacrolimus or $1 \mu \mathrm{M}$ for midazolam) and telaprevir $(1 \mu \mathrm{M}$ for tacrolimus or $0.5 \mu \mathrm{M}$ for midazolam) in HLM were performed after varying durations of pre-incubation with telaprevir $(0,15$, or 30 minutes $)$. Each of the three pre-incubation duration experiments was further designed to assess whether the IC50 shifts observed in earlier experiments were NADPHdependent (i.e., CYP-dependent) and whether the time-dependent IC50 shift was related to CYP3A inactivation (i.e., irreversible or slowly reversible inhibition). For the NADPH-dependent experiment, the incubation protocol was essentially the same as that described for the IC50 shift experiment except that either NADPH or vehicle was added at the outset of pre-incubation; for the vehicle incubation, $1 \mathrm{mM}$ NADPH was added along with the substrate to initiate the reporting reaction. For the reversibility experiment, pre-incubation was conducted with a 20 -fold greater concentration of HLM $(1 \mathrm{mg} / \mathrm{ml})$ at a volume of $500 \mu \mathrm{l}$. After the designated duration of pre-incubation with telaprevir $(0,15$, or $30 \mathrm{~min})$, the concentrated microsomal incubate was diluted 20 -fold (i.e., to $0.05 \mathrm{mg} / \mathrm{ml}$ ). Fifty $\mu \mathrm{l}$ of the diluted, pre-incubation mixture was transferred to a $200 \mu \mathrm{l}$ reaction mixture containing substrate (final concentration $0.5 \mu \mathrm{M}$ tacrolimus or $1 \mu \mathrm{M}$ midazolam) and $1 \mathrm{mM}$ of fresh $\mathrm{NADPH}$, which was allowed to co-incubate for 2 minutes before quenching.

\section{Metabolite Analysis}

1'-OH MDZ and 13-DMT concentrations were quantified using an Agilent 1290 HPLC system (Agilent, Santa Clara, CA) connected to a triple quadrupole mass spectrometer (Agilent 6410 LCMS/MS) operated in the positive ion mode. Chromatography for 1-OH-MDZ in the acetonitrile extract was achieved using a reverse-phase column (Zorbax SB $\mathrm{C}_{18}, 2.1 \mathrm{~mm} \times 150 \mathrm{~mm} \times 5 \mu \mathrm{m}$; (Agilent, Santa Clara, CA) at $35^{\circ} \mathrm{C}$. The aqueous (A) mobile phase consisted of $10 \mathrm{mM}$ ammonium formate $(\mathrm{pH}=4)$ and $100 \%$ acetonitrile was used for the organic (B) mobile phase. The gradient for the mobile phase was as follows: (1) mobile phase A was set to $55 \%$ from 0 to $5 \mathrm{~min}$ at a total flow of $0.25 \mathrm{ml} / \mathrm{min}$, (2) a linear gradient from $5 \mathrm{~min}$ to 6 min decreased phase A to $5 \%$ and increased flow to $0.3 \mathrm{ml} / \mathrm{min}$, which were held until $8 \mathrm{~min}$, (3) a linear gradient increased phase A back to $55 \%$ from 8 to $8.1 \mathrm{~min}$ and decreased total flow back to 0.25 $\mathrm{ml} / \mathrm{min}$, which were maintained for the remainder of the run. The total run time was 12 minutes per sample. 1'-OH-MDZ and $\mathrm{d}_{4}-1-\mathrm{OH}-\mathrm{MDZ}$ were quantified under single reaction monitoring mode using specific precursor/product ion transition. The mass transition for 1'-OH-MDZ was $m / z$ $342 \rightarrow 168.1$ at a collision energy of $40 \mathrm{~V}$. The mass transition for $\mathrm{d}_{4}-1$ ' $-\mathrm{OH}-\mathrm{MDZ}$ was $m / z \quad 346 \rightarrow 168$ at a collision energy of $44 \mathrm{~V}$.

Chromatographic separation of the 13-DMT extract was achieved using a reverse-phase column (Zorbax Eclipse XDB C $8,3 \mathrm{~mm}$ x $150 \mathrm{~mm}$ x $5 \mu \mathrm{m}$; Agilent, Santa Clara, CA) operated at $60^{\circ} \mathrm{C}$. The aqueous (A) mobile phase consisted of $10 \mathrm{mM}$ ammonium formate and $0.1 \mathrm{mM}$ sodium acetate $(\mathrm{pH}=7)$. The organic (B) mobile phase consisted of $100 \%$ methanol with $0.1 \mathrm{mM}$ sodium acetate. The gradient for the mobile phases was: (1) mobile phase A was set to $40 \%$ at $0 \mathrm{~min}$ at a total flow of 
$0.3 \mathrm{ml} / \mathrm{min}$, (2) a linear gradient to 10 minutes decreased phase A to $10 \%$, (3) mobile phase A was increased back to $40 \%$ by a linear gradient from 11 to 11.1 minutes, which was maintained for the remainder of the run. Total run time was 12.5 minutes per sample. 13-DMT and rapamycin were quantified under single reaction monitoring mode using specific precursor/product ion transition. The mass transition for 13-DMT was $\mathrm{m} / \mathrm{z} \quad 812.5 \rightarrow 602.3$ at a collision energy of $36 \mathrm{~V}$. The mass transition for rapamycin was $m / z \quad 936.4 \rightarrow 409.3$ at a collision energy of $60 \mathrm{~V}$.

\section{DATA ANALYSIS}

\section{IC50 Shift Experiment}

An IC50 model, assuming classical MichaelisMenten kinetics, was fitted to the metabolite formation rate data and statistical significance of the shift in IC50 estimates was ascertained by an unpaired t-test using GraphPad Prism Version 5.04 (La Jolla, CA). The observed data points and model predictions were normalized to vehicle control and activity was graphically presented as a fraction of control versus inhibitor concentration plots.

\section{NADPH Dependence and Reversibility Experiment}

The mean data for each of the experiments was normalized to the mean of their respective vehicle control incubations. The control groups used were identical to the co-incubations with telaprevir except that vehicle $(0.3 \%$ DMSO $)$ was added instead of the inhibitor. The two means of the experimental and control groups were assumed to be independent. The standard deviation of the ratio was calculated utilizing a propagation of error equation (22). The resultant means and standard deviations of the ratios for the three incubation groups were then compared ( 0 vs $15 \mathrm{~min}, 0$ vs 30 min, 15 vs $30 \mathrm{~min}$ ) using an unpaired t-test (GraphPad 5.04, La Jolla, CA) with a $\mathrm{p}<0.05$ assigned as statistically significant.

\section{RESULTS}

Model predictions for the various inhibition scenarios precipitated by telaprevir are presented in Table 1, along with the reported magnitude of in vivo interactions with midazolam and telaprevir (5, 12). Our static DDI modeling indicates that competitive inhibition by telaprevir and its diastereomeric metabolite VRT-1237394 cannot fully explain the profound inhibitory interactions observed with either midazolam or tacrolimus. With respect to competitive inhibition of midazolam oral clearance, an approximate 2.6- to 4.2-fold reduction in clearance was predicted for the "least" inhibition scenario. The magnitude of this reduction increased to 3.7-5.2-fold for the "maximum" inhibition scenario. In our "extreme" scenario, where all of the dose is completely absorbed and escapes first-pass extraction, a 5.9- to 7.8-fold reduction in apparent oral clearance of midazolam might be observed. Predictions for all three competitive inhibition scenarios fell short of the 13.5-fold decrease in midazolam oral clearance observed by Garg et al. (12).

For the predicted interaction with tacrolimus, an approximate 3.0 - to 6.2 -fold reduction in oral clearance was predicted in the "least" inhibition scenario. A 9.2- to 14-fold reduction in oral clearance was observed for the "maximum" inhibition scenario, and a 14- to 21 -fold reduction was predicted in the "extreme" scenario where total administered dose is absorbed and escapes first-pass processes. Here again, the predictions of all three competitive inhibition scenarios fell short of the observed interaction of 67-fold reduction in tacrolimus oral clearance (5).

We evaluated telaprevir for time-dependent inhibition (TDI) in vitro. For both substrates, leftward shifts in their IC50 curves were observed following a 30-min pre-incubation with telaprevir (Figure 1) indicating that inhibition of CYP3A is time-dependent. The IC50 for midazolam was lowered from $0.74 \mu \mathrm{M}$ to $0.19 \mu \mathrm{M}$, a 3.8-fold shift that was statistically significant $(\mathrm{p}<0.05)$. Likewise, the IC50 for tacrolimus was lowered from $4.0 \mu \mathrm{M}$ to $0.36 \mu \mathrm{M}$, an 11-fold shift that was also statistically significant. An apparent activation of 13-DMT formation occurred at lower tacrolimus concentrations in the IC50 experiment that was not subjected to pre-incubation. This observation may be explained by allosterism of CYP3A which is not accounted for in the classical IC50 model.

TDI was also demonstrated in the more detailed follow-up study; the results are depicted in Figure 2 . As the pre-incubation duration increased, 1'-OHMDZ formation rate showed a progressive decline provided NADPH was present during preincubation. TDI was absent when pre-incubations were performed in the absence of NADPH. Preincubation with telaprevir also inhibited tacrolimus 
oxidation to 13-DMT in a time-dependent manner contingent upon the presence of NADPH.

In order to assess whether the observed TDI reflected irreversible inactivation of CYP3A, we performed a dilution experiment. A pre-incubation with telaprevir was performed using a 20-fold higher concentration of HLM; the pre-incubated HLM mixture was then diluted 20-fold prior to substrate incubation. The reporter reaction with either midazolam or tacrolimus in the 0 -min telaprevir pre-incubation time-group was comparable to its vehicle control, indicating that competitive inhibition by telaprevir in the diluted microsomal incubate was minimal or nearly absent. In the case of midazolam, dilution effectively reversed the inhibition observed after preincubation with telaprevir, as indicated by the minimal difference $(<20 \%)$ in 1'-OH-MDZ formation rates between zero-time and either 15 or $30 \mathrm{~min}$ of pre-incubation. TDI of tacrolimus oxidation appeared to be more complicated in nature compared to that of midazolam. A reduction in the magnitude of the TDI was indeed observed after dilution; however, a significant component of non-reversible or very slowly reversible inhibition remained. In fact, for the $30 \mathrm{~min}$ pre-incubation, the degree of inhibition observed after dilution hardly differed from that observed in the no-dilution group.

\section{DISCUSSION}

Through the use of established mechanistic static DDI models, we predicted the degree of reduction in oral clearance of two sensitive CYP3A substrates - midazolam and tacrolimus for the scenario when inhibition mechanism was limited to competitive inhibition by the parent drug and its primary metabolite VRT-127394. Our model grossly underestimated the magnitude of telaprevir interactions, even after recognizing the highest possible transient, local inhibitor concentrations in the intestinal mucosa and hepatoportal blood during first-pass. All this was overshadowed by the fact that even when we assumed complete abolition of all first-pass processes $\left(F_{\text {total }}=1\right)$, we still failed to predict the magnitude of the observed DDI. This observation is especially instructive in the case of tacrolimus; abolition of first-pass metabolism would at most result in a 21-fold decrease in oral clearance, which is in contrast to the reported 67fold decrease in a recent healthy human subject study.

\begin{tabular}{|c|c|c|}
\hline Parameter & Midazolam & Tacrolimus \\
\hline$F_{a}$ & $0.90^{*}$ & $0.96^{*}$ \\
\hline$F_{g}$ & $0.51 *$ & $0.14 *$ \\
\hline$F_{g, i n h}($ least $)$ & 0.68 to 0.77 & 0.23 to 0.31 \\
\hline$F_{g, \sinh }^{\prime}(\operatorname{maximum})$ & 0.94 to 0.94 & 0.70 to 0.71 \\
\hline$F_{h}$ & $0.56^{*}$ & $0.97 *$ \\
\hline$F_{h, i n h}($ least $)$ & 0.71 to 0.78 & 0.98 to 0.99 \\
\hline$F_{h, \text { inh }}^{\prime}(\operatorname{maximum})$ & 0.75 to 0.78 & 0.99 to 0.99 \\
\hline$F_{\text {total }}$ & $0.25^{*}$ & $0.13 *$ \\
\hline$F_{\text {total,inh }}($ least $)$ & 0.44 to 0.54 & 0.22 to 0.30 \\
\hline$F_{\text {total,inh }}^{\prime}$ (maximum) & 0.63 to 0.66 & 0.66 to 0.68 \\
\hline$C L_{h}, \mathrm{~L} / \mathrm{h}$ & $39.4^{*}$ & $2.69 *$ \\
\hline$C L_{h, i n h}, \mathrm{~L} / \mathrm{h}$ & 26 to 20 & 1.5 to 0.98 \\
\hline$C L_{\text {oral }}, \mathrm{L} / \mathrm{h}$ & 152 & 20.7 \\
\hline$C L_{\text {oral,inh }}($ least $), \mathrm{L} / \mathrm{h}$ & 59 to 36 & 6.8 to 3.3 \\
\hline$C L_{\text {oral,inh }}$ (maximum), $\mathrm{L} / \mathrm{h}$ & 40 to 29 & 2.3 to 1.5 \\
\hline$C L_{\text {oral }} / C L_{\text {oralinh }}($ least $)$ & 2.6 to 4.2 & 3.0 to 6.2 \\
\hline$C L_{\text {oral }} / C L_{\text {oral,inh }}$ (maximum) & 3.7 to 5.2 & 9.2 to 14 \\
\hline$C L_{\text {oral }} / C L_{h, \text { inh }}$ (1st pass abolished) & 5.9 to 7.8 & 14 to 21 \\
\hline Observed $C L_{\text {oral }} / C L_{\text {oral,inh }}$ & $13.5^{*}$ & $66.7^{*}$ \\
\hline
\end{tabular}

Table 1. Summary of Static DDI Model Predictions. *denotes value taken directly or inferred from the literature $(5,12-$ 13, 18); (least) pertains to the scenario governed by purely systemic inhibitor concentrations; (maximum) pertains to the scenario featuring elevated local concentrations at sites of first-pass elimination; (abolished) pertains to the "extreme" scenario where first-pass metabolism is completely abolished. 


\section{1'-OH-Midazolam Formation}

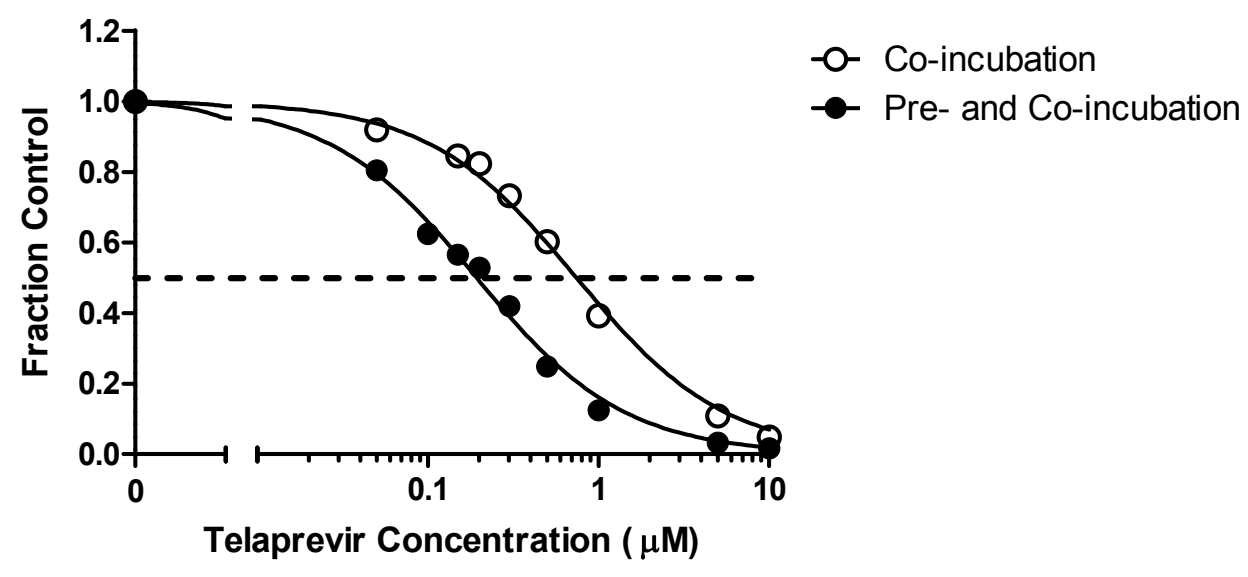

13-DMT Formation

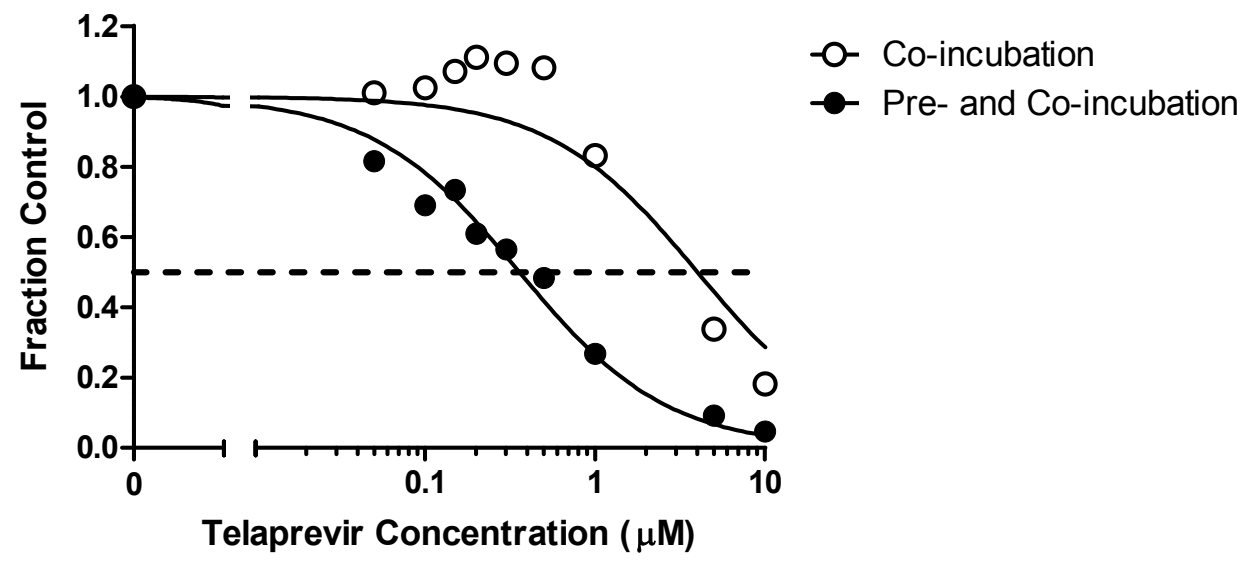

Figure. 1. Comparison of telaprevir IC50 curve for the co-incubation experiment ( $(\circ)$ versus pre- and co-incubation experiment (•) for midazolam (top) and tacrolimus (bottom). The solid line represents the fit to an IC50 model.

Collectively, the competitive inhibition model suggests that 1) profound inhibition must occur during both first-pass and systemic phase of drug clearance; 2) the degree of competitive inhibition estimated based upon the referenced in vitro $K_{i}$ is either inaccurate (i.e., in vivo $K_{i}$ differ drastically from in vitro $K_{i}$ ) or presence of other mechanisms of inhibition must be considered; and 3) the mechanism of telaprevir-precipitated interactions is complicated by substrate-dependence (i.e., possibly explained by some sort of reciprocal substrateinhibitor interaction at the CYP3A binding sites).

There have been reports of putative MBI involving the parent telaprevir. While we were able to confirm the existence of TDI, it was observed to be partially or fully reversible depending on the substrate selected. This reversibility, particularly with midazolam as the CYP3A substrate, casts doubt on parent telaprevir as a mechanism-based inhibitor, and points to the possible formation of potent, reversibly acting inhibitory metabolites during pre-incubation. Vertex has reported that telaprevir can spontaneously convert to VRT127394 in circulation; however, we were not able to detect the presence of this diastereomeric metabolite in our incubations without the availability of an authentic standard. We do not believe the formation of VRT-127394 can easily explain the remarkable IC50 shift following preincubation since the competitive $K_{i}$ of VRT-127394 
is about equal to that of telaprevir (6). Similar in vitro findings of reversible TDI have been reported with another first-generation $\mathrm{HCV}$ protease inhibitor, boceprevir (23). Furthermore, observations of reversible TDI involving cytochrome $\mathrm{P} 450 \mathrm{~s}$ is not limited to the $\mathrm{HCV}$ protease inhibitors. Reversibility in TDI of cytochrome P450s has been reported for both $R$ fluoxetine and amiodarone (24), both of which are known to form inhibitory metabolites. Due to the potential for TDI to be of a reversible nature, the importance of incorporating a "dilution" step in experiments to measure the kinetics of enzyme inactivation cannot be overstated. In their recent in vitro study of telaprevir's inhibition of tacrolimus metabolism (7), Oda and Yamano failed to mention the inclusion of a dilution step (or any other means to eliminate the carryover of telaprevir from the pre-incubation) in their MBI experiment for generating estimates of enzyme inactivation parameters $k_{\text {inact }}$ and $K_{I}$. As a result, their model predictions of the in vivo DDI between telaprevir and tacrolimus based on the assumption of MBI of CYP3A by parent telaprevir may not be valid. Until we ascertain the exact nature of the TDI, attempts at in vitro-to-in vivo scaling of drug interactions with telaprevir involving CYP3A are premature.

\section{1'-OH-Midazolam Formation}

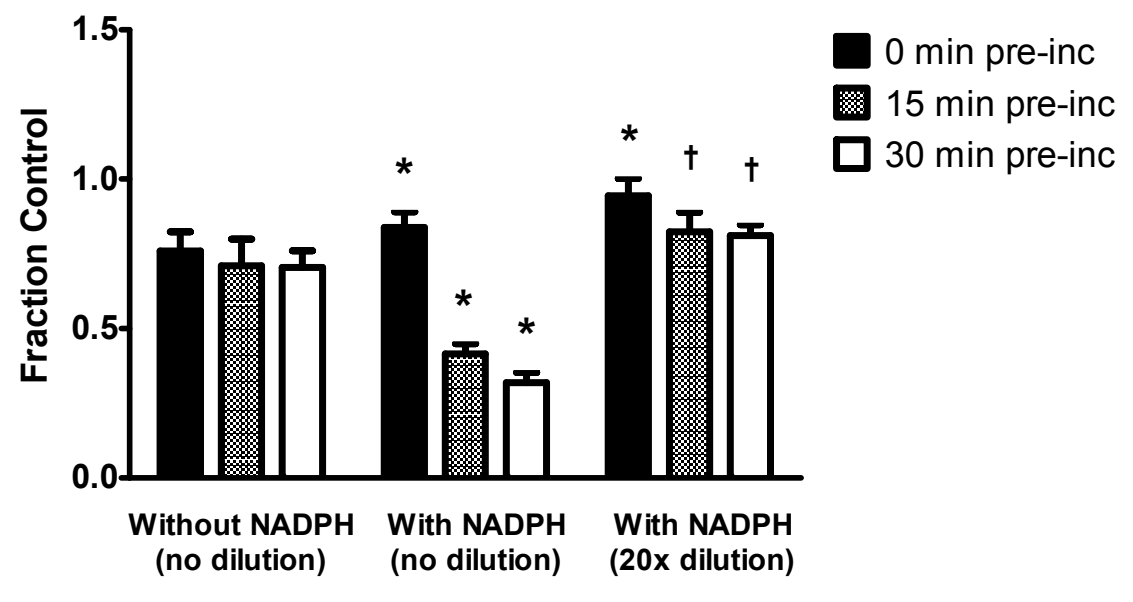

13-DMT Formation

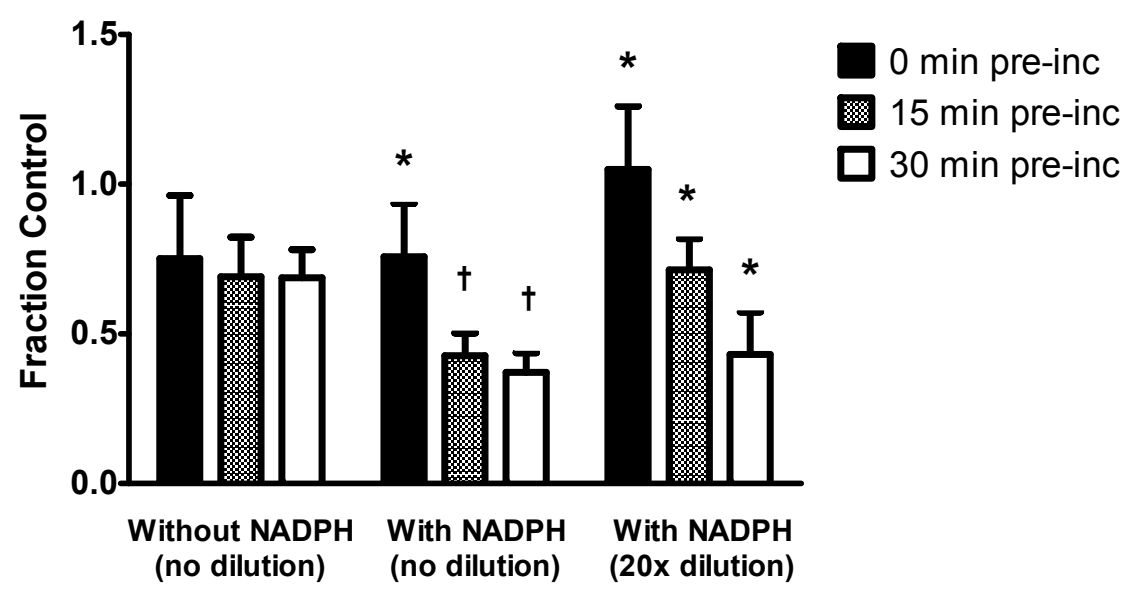

Figure 2. Determination of NADPH-dependence and reversibility of time-dependent inhibition of 1'-OH-MDZ formation (top) and 13-DMT formation (bottom) by telaprevir. Error bars represent standard deviation. * denotes statistically significant difference $(\mathrm{p}<0.05)$ between the mean of this time point and those of the other time points within a treatment group. $\dagger$ denotes statistically significant difference $(\mathrm{p}<0.05)$ between the mean of this time point and mean of the zerotime pre-incubations within a treatment group. 
From a general perspective, the distinction between reversible and irreversible TDI has important clinical implications in regards to the outcomes of DDI. In the case of an irreversible TDI, such as MBI, the time to reach maximum inhibition following addition of the inhibitor and the time it takes for inhibition to dissipate following withdrawal of the inhibitor depend on both the elimination half-life of the inhibitor as well as the turnover half-life of the enzyme. If the inhibitor has a relatively short half-life as in the case for telaprevir $(<24 \mathrm{hrs})$, the turnover of the CYP3A protein in the intestinal mucosa and the liver becomes the rate-limiting process. In contrast, if the TDI is reversible and attributable to the formation of an equally or more potent inhibitory metabolite, the dynamics of inhibition during or post inhibitor treatment will depend on either the elimination halflife of the parent drug if the washout kinetics of the inhibitory metabolite is formation-rate-limited, or the elimination half-life of the inhibitory metabolite if that is rate-limiting for the washout of the metabolite. Hence, the conclusion of an MBI should not be based solely on the observation of TDI in an IC50 shift study; assessment of irreversibility in inhibition should always be the critical test of a MBI.

Finally, it is important to recognize that telaprevir could precipitate DDI via non-CYP3A related mechanisms. Specifically, telaprevir is known to inhibit intestinal P-glycoprotein, which has been shown to result in increased in digoxin absorption (12). It is conceivable that the profound in vivo interaction between telaprevir and tacrolimus stems from the simultaneous inhibition of intestinal CYP3A and P-glycoprotein, disrupting the interplay between CYP3A and P-glycoprotein at the intestinal mucosa (25). However, it should be noted that a complete halt to intestinal metabolism and efflux transport processes still cannot fully account for the magnitude of the observed DDI, as illustrated by the under-prediction of our first-pass "abolition" modeling scenario. Interplay between CYP3A and P-glycoprotein has also been suggested to occur at the bile canaliculi, which may play a role in the hepatic clearance of tacrolimus (26). Dual-inhibition of CYP3A and P-glycoprotein at the bile canaliculi could have an impact on both first-pass and systemic extraction of tacrolimus by the liver. It is also worth noting that tacrolimus itself has been associated with time-dependent inhibition of CYP3A (27). Modulation in such tacrolimus-mediated inhibitory processes could contribute to the magnitude of the observed DDI. The apparent activation of 13-DMT formation seen at low telaprevir concentrations (Figure 1) hints at allosteric effects, which could add further complexity to the kinetics of this interaction. Further investigation of the time- or metabolismdependent inhibition of CYP3A seems worthwhile as it might unveil unusual or novel mechanisms of metabolic inhibition for this set of remarkable CYP3A-based DDIs.

\section{ACKNOWLEDGEMENTS}

This research was supported by the National Institutes of Health, National Institute of General Medical Sciences (P01 GM32165); and the University of Washington School of Pharmacy Drug Metabolism, Pharmacokinetics, and Transport Research Program. BC is a recipient of the William E. Bradley Fellowship.

\section{REFERENCES}

1. Ghany, M. G.; Nelson, D. R.; Strader, D. B.; Thomas, D. L.; Seeff, L. B., An update on treatment of genotype 1 chronic hepatitis $\mathrm{C}$ virus infection: 2011 practice guideline by the American Association for the Study of Liver Diseases. Hepatology (Baltimore, Md.) 2011, 54 (4), 1433-44.

2. Manos, M. M.; Shvachko, V. A.; Murphy, R. C.; Arduino, J. M.; Shire, N. J., Distribution of hepatitis $\mathrm{C}$ virus genotypes in a diverse US integrated health care population. Journal of medical virology 2012, 84 (11), 1744-50.

3. Talavera Pons, S.; Lamblin, G.; Boyer, A.; Sautou, V.; Abergel, A., Drug interactions and protease inhibitors used in the treatment of hepatitis $\mathrm{C}$ : how to manage? European journal of clinical pharmacology 2014, 70 (7), 775-89.

4. Coilly, A.; Roche, B.; Dumortier, J.; Leroy, V.; Botta-Fridlund, D.; Radenne, S.; Pageaux, G. P.; SiAhmed, S. N.; Guillaud, O.; Antonini, T. M.; HaimBoukobza, S.; Roque-Afonso, A. M.; Samuel, D.; Duclos-Vallee, J. C., Safety and efficacy of protease inhibitors to treat hepatitis $\mathrm{C}$ after liver transplantation: a multicenter experience. Journal of hepatology 2014, 60 (1), 78-86.

5. Garg, V.; van Heeswijk, R.; Lee, J. E.; Alves, K.; Nadkarni, P.; Luo, X., Effect of telaprevir on the pharmacokinetics of cyclosporine and tacrolimus. Hepatology (Baltimore, Md.) 2011, 54 (1), 20-7.

6. U.S. Food and Drug Administration Center for Drug Evaluation and Research. Telaprevir Clinical Pharmacology and Biopharmaceutics Review(s). 
http://www.accessdata.fda.gov/drugsatfda_docs/nda/ 2011/201917Orig1s000ClinPharmR.pdf.

7. Oda, K.; Yamano, K., Effect of telaprevir on the metabolism and hepatic uptake of tacrolimus (FK506). Biopharmaceutics \& drug disposition 2014, 35 (9), 501-12.

8. Zheng, S.; Easterling, T. R.; Umans, J. G.; Miodovnik, M.; Calamia, J. C.; Thummel, K. E.; Shen, D. D.; Davis, C. L.; Hebert, M. F., Pharmacokinetics of tacrolimus during pregnancy. Therapeutic drug monitoring 2012, 34 (6), 660-70.

9. Obach, R. S.; Walsky, R. L.; Venkatakrishnan, K.; Gaman, E. A.; Houston, J. B.; Tremaine, L. M., The utility of in vitro cytochrome P450 inhibition data in the prediction of drug-drug interactions. The Journal of pharmacology and experimental therapeutics 2006, 316 (1), 336-48.

10. Hisaka, A.; Ohno, Y.; Yamamoto, T.; Suzuki, H., Theoretical considerations on quantitative prediction of drug-drug interactions. Drug metabolism and pharmacokinetics 2010, 25 (1), 48-61.

11. Galetin, A.; Gertz, M.; Houston, J. B., Potential role of intestinal first-pass metabolism in the prediction of drug-drug interactions. Expert opinion on drug metabolism \& toxicology 2008, 4 (7), 909-22.

12. Garg, V.; Chandorkar, G.; Farmer, H. F.; Smith, F.; Alves, K.; van Heeswijk, R. P., Effect of telaprevir on the pharmacokinetics of midazolam and digoxin. Journal of clinical pharmacology 2012, 52 (10), 1566-73.

13. Gertz, M.; Houston, J. B.; Galetin, A., Physiologically based pharmacokinetic modeling of intestinal first-pass metabolism of CYP3A substrates with high intestinal extraction. Drug metabolism and disposition: the biological fate of chemicals 2011, 39 (9), 1633-42.

14. Gorski, J. C.; Jones, D. R.; Haehner-Daniels, B. D.; Hamman, M. A.; O'Mara, E. M., Jr.; Hall, S. D., The contribution of intestinal and hepatic CYP3A to the interaction between midazolam and clarithromycin. Clinical pharmacology and therapeutics 1998, 64 (2), 133-43.

15. Rowland, M.; Benet, L. Z.; Graham, G. G., Clearance concepts in pharmacokinetics. Journal of pharmacokinetics and biopharmaceutics 1973, 1 (2), 123-36.

16. Lu, C.; Hatsis, P.; Berg, C.; Lee, F. W.; Balani, S. K., Prediction of pharmacokinetic drug-drug interactions using human hepatocyte suspension in plasma and cytochrome P450 phenotypic data. II. In vitro-in vivo correlation with ketoconazole. Drug metabolism and disposition: the biological fate of chemicals 2008, 36 (7), 1255-60.

17. Paine, M. F.; Hart, H. L.; Ludington, S. S.; Haining, R. L.; Rettie, A. E.; Zeldin, D. C., The human intestinal cytochrome P450 "pie". Drug metabolism and disposition: the biological fate of chemicals 2006, 34 (5), 880-6.

18. Heizmann, P.; Ziegler, W. H., Excretion and metabolism of 14C-midazolam in humans following oral dosing. Arzneimittel-Forschung 1981, 31 (12a), 2220-3.

19. Yang, J.; Jamei, M.; Yeo, K. R.; Tucker, G. T.; Rostami-Hodjegan, A., Prediction of intestinal firstpass drug metabolism. Current drug metabolism 2007, 8 (7), 676-84.

20. Dai, Y.; Hebert, M. F.; Isoherranen, N.; Davis, C. L.; Marsh, C.; Shen, D. D.; Thummel, K. E., Effect of CYP3A5 polymorphism on tacrolimus metabolic clearance in vitro. Drug metabolism and disposition: the biological fate of chemicals 2006, 34 (5), 83647.

21. Kamdem, L. K.; Streit, F.; Zanger, U. M.; Brockmoller, J.; Oellerich, M.; Armstrong, V. W.; Wojnowski, L., Contribution of CYP3A5 to the in vitro hepatic clearance of tacrolimus. Clinical chemistry 2005, 51 (8), 1374-81.

22. Reckhow, D. Chapter XXIII: Parametric Statistics. http://www.ecs.umass.edu/cee/reckhow/courses/572/ 572bk23/572BK23.html (accessed July 7, 2014).

23. Chu, X.; Cai, X.; Cui, D.; Tang, C.; Ghosal, A.; Chan, G.; Green, M. D.; Kuo, Y.; Liang, Y.; Maciolek, C. M.; Palamanda, J.; Evers, R.; Prueksaritanont, T., In vitro assessment of drug-drug interaction potential of boceprevir associated with drug metabolizing enzymes and transporters. Drug metabolism and disposition: the biological fate of chemicals 2013, 41 (3), 668-81.

24. Lee, J. Y.; Lee, S. Y.; Oh, S. J.; Lee, K. H.; Jung, Y. S.; Kim, S. K., Assessment of drug-drug interactions caused by metabolism-dependent cytochrome P450 inhibition. Chemico-biological interactions 2012, 198 (1-3), 49-56.

25. Cummins, C. L.; Jacobsen, W.; Benet, L. Z., Unmasking the dynamic interplay between intestinal P-glycoprotein and CYP3A4. The Journal of pharmacology and experimental therapeutics 2002, 300 (3), 1036-45.

26. Wu, C. Y.; Benet, L. Z., Disposition of tacrolimus in isolated perfused rat liver: influence of troleandomycin, cyclosporine, and gg918. Drug metabolism and disposition: the biological fate of chemicals 2003, 31 (11), 1292-5.

27. Amundsen, R.; Asberg, A.; Ohm, I. K.; Christensen, H., Cyclosporine A- and tacrolimus-mediated inhibition of CYP3A4 and CYP3A5 in vitro. Drug metabolism and disposition: the biological fate of chemicals 2012, 40 (4), 655-61. 